\title{
Evaluating measurement accuracy and repeatability with a new device that records spatial knee movement
}

\author{
FEI TIAN ${ }^{1,2}$, QIUYUE HUANG ${ }^{1}$, ZHI ZHENG ${ }^{1,3}$, NINGWEI LI ${ }^{1}$, \\ TiNG ZHU ${ }^{1}$, WeI DAI ${ }^{1}$, SHAOBAI WANG ${ }^{1}$ * \\ ${ }^{1}$ School of Kinesiology, Key Laboratory of Exercise and Health Sciences of Ministry of Education, \\ Shanghai University of Sport, Shanghai, China. \\ ${ }^{2}$ Department of Rehabilitation Medicine, Heping Hospital Affiliated to Changzhi Medical College, Shanxi, China. \\ ${ }^{3}$ Department of Sports Medicine, Shanghai Sixth People's Hospital, Shanghai, China.
}

\begin{abstract}
Purpose: A novel portable system has been used to evaluate spatial knee movement, but its accuracy and repeatability is not known. The aim of this study was to investigate the accuracy and repeatability of the measurement. Methods: Ten healthy participants were included, and the knee motion trajectory during walking were assessed. Six evaluations were conducted (three days by two raters) for each participant. The motion parameters at the key points and the range of motion were statistically analyzed. Intraclass correlation coefficients (ICC), standard error of the measurement (SEM), and the Bland-Altman method were used. Results: For intra-rater repeatability, 1) the ICC values range from 0.75 to 0.9 for rotations; and 0.64 to 0.96 for translations. Among the ICC values, $100 \%$ of rotations and $90 \%$ of translations were not less than $0.70 ; 2$ ) among the SEM values, $100 \%$ of rotations were not more than $5^{\circ}$, while $73.3 \%$ of translations were less than $3 \mathrm{~mm}$. For inter-rater repeatability, 1) the ICC values range from 0.68 to 0.99 for rotations; and 0.57 to 0.93 for translations. Among the ICC values, $95.6 \%$ of rotations and $82.2 \%$ of translations were not less than $0.70 ; 2$ ) among the SEM values, $100 \%$ of rotations were not more than $5^{\circ}$, and $48.9 \%$ of translations were less than $3 \mathrm{~mm}$. The Bland-Altman plots showed good agreement for intra- and inter-repeatability. Conclusions: The results indicated that the accuracy and repeatability of the measurement were acceptable, except for the inter-rater repeatability for translation. This may help researchers and physicians better interpret the measurement data.
\end{abstract}

Key words: gait analysis, repeatability, motion analysis, three-dimensional, knee joint

\section{Introduction}

Knee joint is one of the most common injury sites, and involves a complex six degrees of freedom (6DOF) coupling motion [7], [8], [13], including flexion-extension (FE), internal-external (IE), and varusvalgus (VV) rotation, as well as anterior-posterior (AP), medial-lateral (ML), and distal-proximal (DP) translation of the tibia with respect to the femur. Abnormal motion trajectory of the knee joint is associated with knee disease and its severity [23]. For example, varus thrust is associated with iliotibial band syndrome and knee osteoarthritis [3], [20]. An increase in anterior translation of the tibia often implies the possibility of anterior cruciate ligament rupture [26]. A reliable and detailed understanding on the motion trajectory of the knee could help clinicians make a constructive decision for knee diseases [8], [24]. Therefore, it is crucial to provide a repeatable and comprehensive measurement.

Majority of motion capture systems (MCS) have not been feasible in clinics. First, the large optical MCS could evaluate three-dimensional (3D) rotation of the knee joint without capability to distinguish the translation, like Vicon and Qualisys [1], [11]. However,

\footnotetext{
* Corresponding author: Shaobai Wang, School of Kinesiology, Key Laboratory of Exercise and Health Sciences of Ministry of Education, Shanghai University of Sport, Shanghai, China. E-mail: wangs@innomotion.biz

Received: February 12th, 2020

Accepted for publication: May 18th, 2020
} 
almost all of previous studies reported only rotation repeatability [1], [10], [11], and only one paper reported anteroposterior repeatability in addition to rotation repeatability [9]. Second, as the gold standard method, biplane fluoroscopy could quantify skeletal movement precisely without the soft tissue artifacts [13]. However, despite biplane fluoroscopy and the large MCS, the applications have been severely limited for clinicians and researchers because of considerable financial and spatial-temporal requirements as well as technical difficulties, particularly in developing countries. No study has revealed the repeatability of in vivo 6-DOF motion trajectory of the knee, which remains a challenge in biomechanics [13]. Therefore, it is essential to address the limitations and develop a novel portable system to comprehensively evaluate the 6-DOF motion trajectory of the knee.

A novel, portable MCS was recently developed and employed in sports medicine to assess the 6-DOF motion trajectory of the knee [22], [26]. However, the accuracy and repeatability of the measurement remain unclear. Using the MCS, manual positioning of anatomical landmarks and the rigid bodies with the infrared reflective markers was still required, which was one of the main sources of measurement errors according to previous studies [11], [19]. Moreover, malpositioning of anatomical landmarks may cause improper definition of the segment coordinate system, which might lead to false results of joint motion trajectory [6], [19]. Moreover, the variability between days and raters could affect the clinical or surgical outcomes over the treatment time [6]. Furthermore, the novel portable MCS has been increasingly applied in sports medicine to detect potential injury risk and abnormal motion for anterior cruciate ligament deficiency and osteoarthritis [25], [26]. Thus, it is crucial to observe the accuracy and testretest repeatability between days and raters to the interpret the findings reasonably. It could be meaningful for physicians to make a constructive decision for knee disease [6], [16].

Therefore, the purpose of this study was to observe the accuracy and repeatability of 6-DOF motion trajectory of the knee on three different days by two raters in healthy participants. We hypothesized that, based on the portable MCS, the accuracy and repeatability of 6-DOF motion trajectory of the knee would be acceptable. The intra-rater repeatability (between days) may be higher than the interrater repeatability (between raters), and the repeatability for knee rotation may be better than for translation.

\section{Materials and methods}

\subsection{Participants}

A total of 10 healthy participants (six males and four females, mean age of $21.8 \pm 1.7$ years, mean height of $173.6 \pm 9.1 \mathrm{~cm}$, and mean weight of $71 \pm 10.1 \mathrm{~kg}$ ) were recruited for this study. All participants were free of any musculoskeletal disorders or chronic systemic diseases. This study was approved by the Ethics Committee of Shanghai University of Sport (2017042). All participants provided written informed consent before the experiment.

\subsection{Experimental protocol and instrumentation}

The 6-DOF motion trajectories of the knee were acquired on three different days, two days apart. For each day, the knee assessments were performed by two raters who had equal training experience using the system. The difference between them was their educational background. One was a physiotherapist, while the other was not. For all participants, the knee was evaluated in the preferred kicking leg [2]. Thus, a total of six assessments were conducted for each participant. Each assessment took about 10-15 minutes. All of the motion trajectory evaluations on the knee were based on the portable MCS (Opti_Knee, Innomotion $^{\circledR}$, Shanghai, China).

The required spatial dimension was approximately $4.0 \times 2.0 \times 2.5 \mathrm{~m}^{3}$. The measurement environment and the composition of the portable MCS are shown in Fig. 1. The main components included a stereo binocular infrared camera (NDI Polaris Spectra, Northern Digital Inc., Waterloo, Canada), a high-speed camera (Basler aca 640-90uc, Basler AG, Ahrensburg, Germany), and an integrated customized software computer (KneeMo V1.0; Innomotion). The attachments consisted of two rigid bodies and one digitizing probe (OK_Marquer, Innomotion), all of which had four infrared reflective markers embedded. The MCS camera captured the knee motion with the frequency of 60 $\mathrm{Hz}$ and tracking accuracy of $0.3 \mathrm{~mm}$ root mean square (RMS). The Basler camera recorded the walking videos. The computer could calculate the 6-DOF motion trajectory of the knee in real time during the collecting process. Additionally, a treadmill (Bipace, Innomotion) was used for walking in this study. 


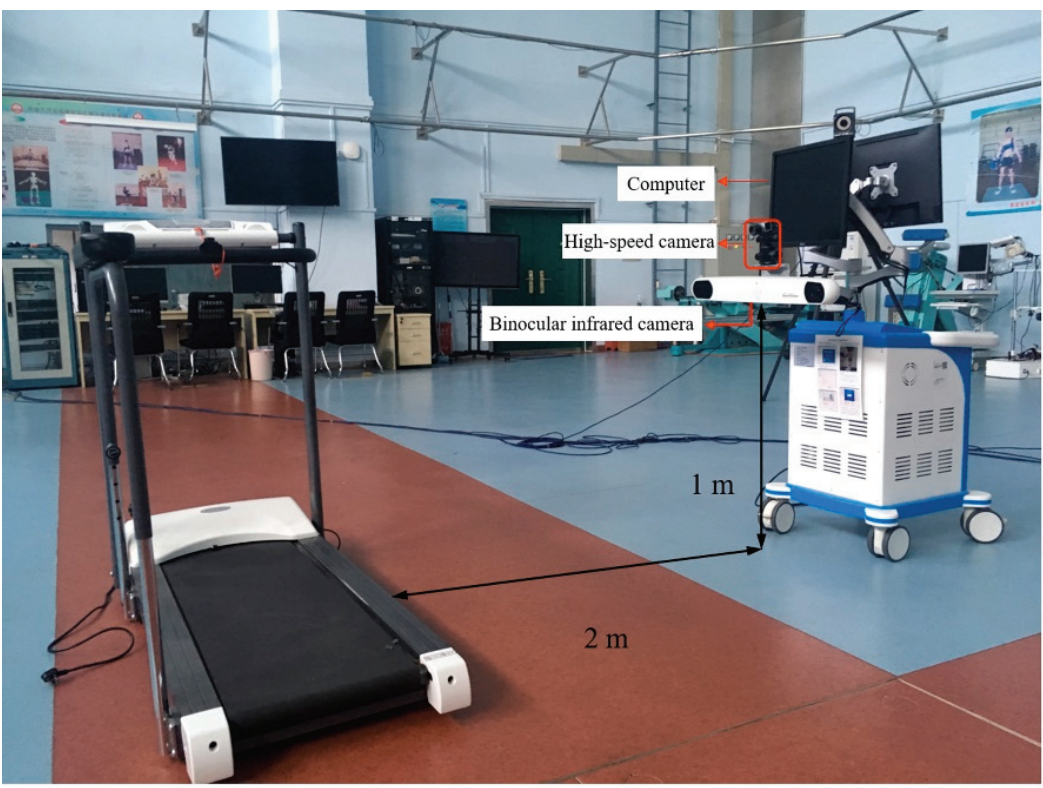

(a)

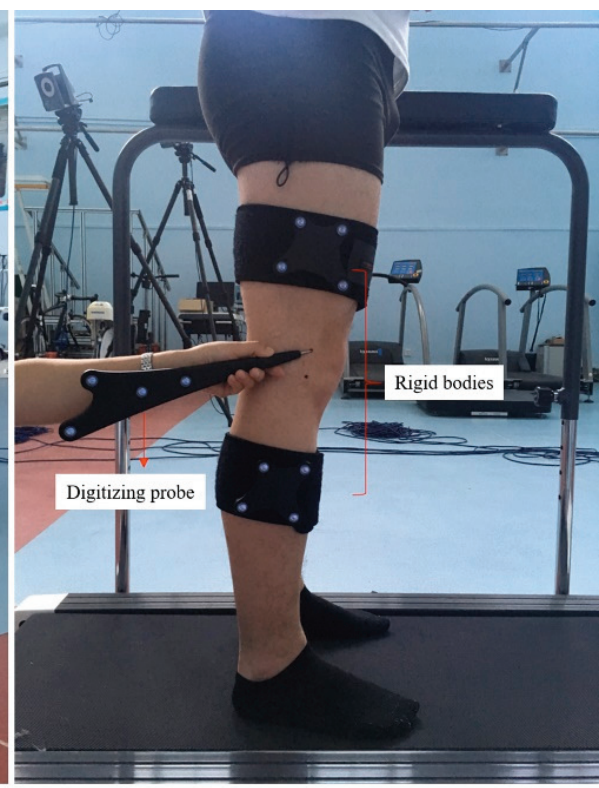

(b)

Fig. 1. The main components (a) and attachments (b) of the portable, optical motion capture system (MCS)

The detailed process of knee motion capture involved the establishment of knee local coordinates and the collection of knee motion trajectory. First, the participants stood naturally (feet shoulder-width apart, toes forward) with two rigid bodies attached on their thigh and shin by Velcro straps (Fig. 1(b)). Second, the digitizing probe was held at the anatomical landmarks of the femur and tibia (including greater trochanter, lateral epicondyle, medial epicondyle, lateral plateau, medial plateau, medial malleolus, and lateral malleolus) to acquire the spatial positions of the anatomical virtual landmarks relative to the infrared markers. Thus, the 3D coordinate system of the tibia with respect to the femur was established (Fig. 2). The origin of the femoral system was defined as the midpoint of the lateral and medial epicondyle of the femur. The line of the lateral and medial epicondyle of the femur was the lateral-medial axis. The anterior-posterior axis was perpendicular to the plane formed by the lateral and medial epicondyle of the femur and the greater trochanter, while the proximal-distal axis was perpendicular to the other two axes. The origin of the tibial system was defined as the midpoint of the medial and lateral tibial plateaus. The lateral-medial axis was the line connecting the medial and lateral tibia plateaus. The anterior-posterior axis was perpendicular to the plane formed by the lateralmedial axis and medial malleolus. The proximal-distal axis was perpendicular to the other two axes. The static position data were recorded as reference base values. Third, no less than 10 continuous gait cycles for $\sim 15 \mathrm{~s}$ at $3 \mathrm{~km} / \mathrm{h}$ for each patient were collected, which was recommended to decrease the motion trajectory vari- ability [17]. A 6-8-minute period of treadmill walking was performed to adapt to the treadmill before the data collection of knee motion trajectory [15]. All of the evaluations were carried out following the same procedure.

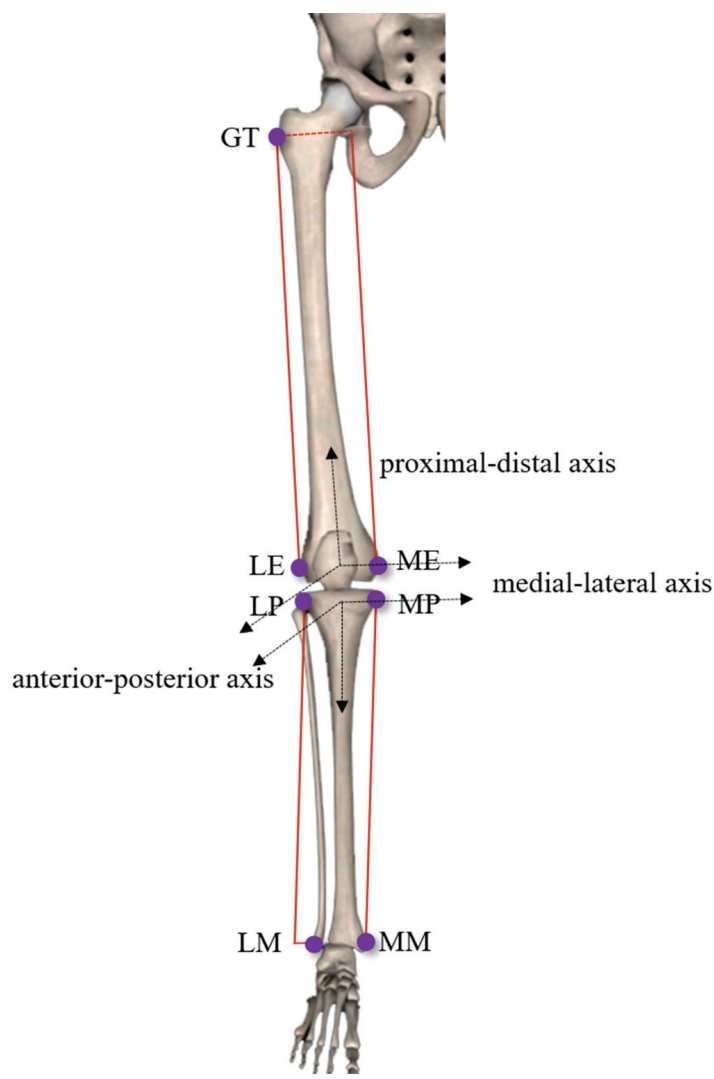

Fig. 2. The establishment of three-dimensional coordinates of the tibia with respect to the femur 


\subsection{Data analysis}

The 6-DOF data of the knee motion trajectory during gait were standardized to 100 points $(0-100 \%$ heel strike) per gait cycle. For each participant, the ensemble averaged curves of knee motion trajectory were calculated for analysis. Similarly to previous studies [1], [22], the motion parameters at four key points (initial contact (IC), peak knee flexion during stance (STPF), peak knee extension during stance (STPE), and peak flexion during swing, (SWPF)) and ROM during the gait cycle were chosen for the statistical analysis.

\subsection{Statistical analysis}

Statistical analysis was performed using SPSS software (version 22.0, SPSS Inc., IBM, Armonk, NY, USA). All parameters on the motion trajectory of the knee were examined for normality using the Shapiro -Wilk test. Intraclass correlation coefficients $\left(\mathrm{ICC}_{2,1}\right)$, standard error of the measurement (SEM), and the Bland-Altman method were performed to evaluate the intra- and inter-rater repeatability of this portable, optical MCS. The intra-rater repeatability indicated agreement between three different days by the same rater, which was determined by an average of two raters. The interrater repeatability denoted agreement between the two raters, which was determined by the average of the three different days.

The $\mathrm{ICC}_{2,1}$ value based on a single measure, absolute agreement, and two-way random effects model, which implied that both people and measurement effects were randomized. According to Cicchetti et al. [5], the $\mathrm{ICC}_{2,1}$ value was rated as follows: 0.00 to 0.39 - poor, 0.40 to 0.59 - fair, 0.60 to 0.74 - good, and 0.75 to 1.00 - excellent. The ICC value of $\geq 0.70$ was usually considered acceptable [4].

SEM investigated the absolute repeatability and provide information on the measurement accuracy or variability of repeated measurements. The equation was $\mathrm{SEM}=\mathrm{s} \sqrt{1-\mathrm{ICC}}$, where $\mathrm{s}$ represented the standard deviation of the sample, and ICC represented the corresponding intraclass correlation coefficient. A lower SEM indicated smaller system error and better reproducibility of the measure. For rotation, the SEM value was considered excellent for angle $<3^{\circ}$, and acceptable for angle $<5^{\circ}[16]$. For translation, according to a repeatability study on KT-2000, a $<3 \mathrm{~mm}$ side-to-side difference was recommended to indicate stable knees [18]. Thereby, the SEM value was considered acceptable $(<3 \mathrm{~mm})$ in the current study.
The Bland-Altman method with 95\% limits of agreement (LoA) was used to evaluate the agreement between different raters and days. According to Teufl et. al. [21], the agreement of 6-DOF motion curves of the knee was conducted during the normalized gait cycle. Additionally, the agreement for ROM in the 6-DOF motion trajectory of the knee was also analyzed by the Bland-Altman method.

\section{Results}

\subsection{Description of the 6-DOF motion trajectory of the knee during gait}

The 6-DOF motion trajectory curves of the knee during gait cycles on three different days by both raters are shown in Fig. 3. The 6-DOF motion trajectory curves of the knee looked nearly overlapped, especially in the FE direction. The 6-DOF motion trajectory of the knee at the IC, STPF, STPE, and SWPF, as well as the ROM during gait were expressed as means (SD) (Table 1).

\subsection{Intra-rater repeatability}

The $\mathrm{ICC}_{\text {Intra-R }}$ values revealed good to excellent correlations (Figs. 4a, 5a). Out of 60 ICC values, $30(100 \%)$ for rotations and $27(90 \%)$ for translations showed a value $\geq 0.70$; while $3(10 \%)$ were $0.64,0.66$, and 0.69 for intra-rater2 for translations. For the 6-DOF motion trajectory of the knee at IC, STPF, STPE, and SWPF, the mean $\mathrm{ICC}_{\text {Intra-R }}$ values were $0.89,0.85$, and 0.94 for FE, IE, and VV rotations, respectively, and $0.80,0.78$, and 0.95 for AP, ML, and DP translations, respectively (Fig. 4a). For the ROM of 6-DOF motion trajectory of the knee during the gait cycle, the mean ICC $_{\text {Intra-R }}$ values were $0.95,0.89$, and 0.82 for FE, IE, and VV rotations, respectively, and $0.88,0.92$, and 0.85 for AP, ML, and DP translations, respectively (Fig. 5a).

The $\mathrm{SEM}_{\text {Intra } \mathrm{R}}$ values are shown in Fig. 4(b) and 5(b) to illustrate the intra-rater absolute repeatability. Out of 60 SEM values, $30(100 \%)$ for rotations and $28(93.3 \%)$ for translations showed values of $\leq 5^{\circ}$ or $5 \mathrm{~mm} ; 2(6.7 \%)$ were $5.01 \mathrm{~mm}$ and $5.21 \mathrm{~mm}$ for intra-rater 2 for AP translation; $26(86.7 \%)$ for rotations and $22(73.3 \%)$ for translations showed values of $<3^{\circ}$ or $3 \mathrm{~mm}$. For the 6-DOF motion trajectory of the knee at IC, STPF, 

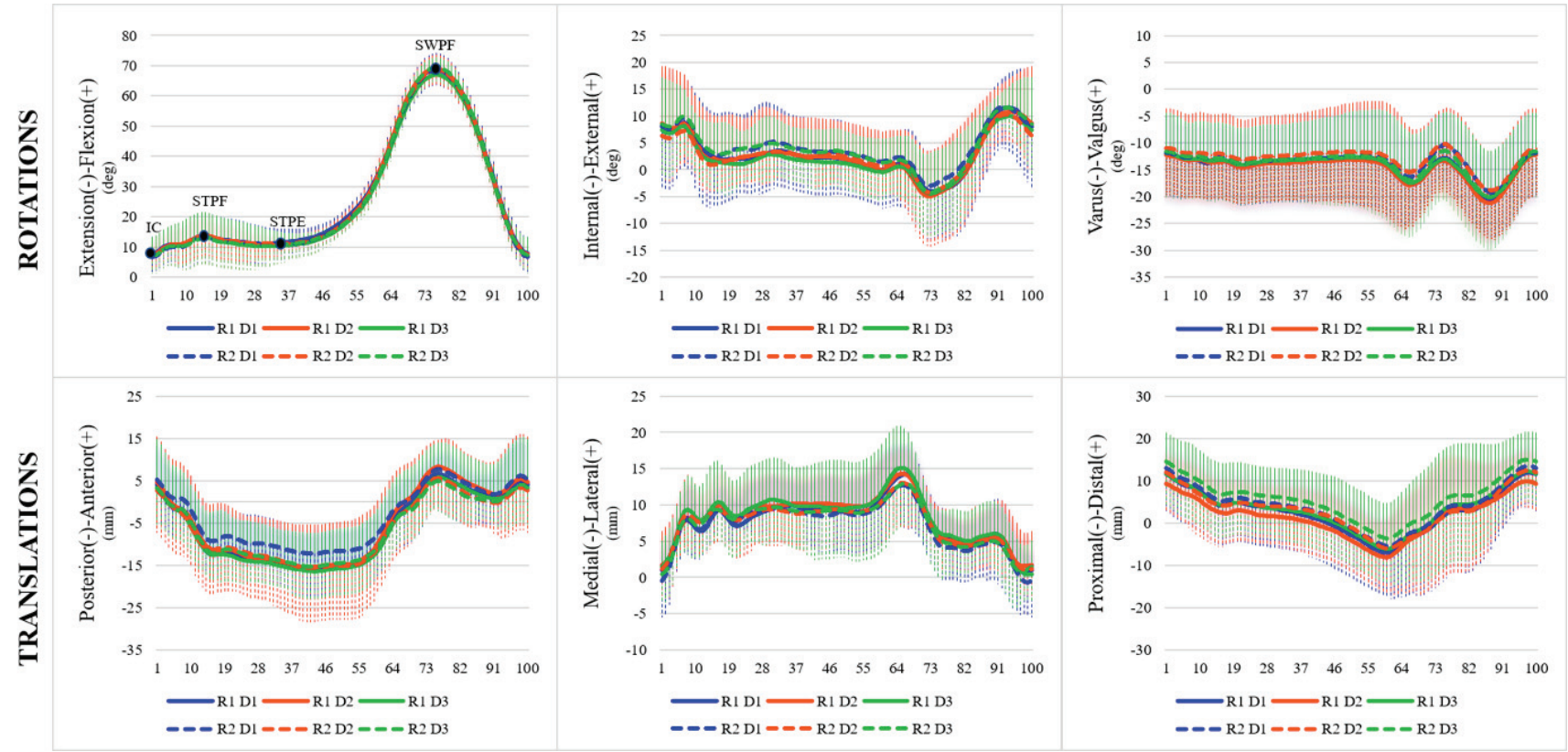

Fig. 3. The 6-DOF curves of knee motion trajectory on three different days by rater1 and rater2. The blue, red, and green refer to the motion parameters on day1, day2, and day3, respectively. The solid curves and the lines above them represent the mean and SD (variability of these cycles) of the gait cycle by rater1, while the dashed curves and the lines below those by rater 2 .

Thus, R1D1 refers to the evaluation on day1 by rater1; R1D2 refers to the evaluation on day 2 by rater1 and so on. The four dots in the extension-flexion curves represent IC, STPF, STPE, and SWPF, respectively

Table 1 . The motion trajectory of the knee at the key points and ROM in 6-DOF during gait

\begin{tabular}{|c|c|c|c|c|c|c|c|}
\hline \multicolumn{2}{|c|}{ Variables } & FE & IE & VV & $\mathrm{AP}$ & ML & $\mathrm{DP}$ \\
\hline \multicolumn{2}{|c|}{1} & 2 & 3 & 4 & 5 & 6 & 7 \\
\hline \multirow{6}{*}{ IC } & R1D1 & $7.86(5.47)$ & $7.95(10.80)$ & $-12.02(7.76)$ & $4.03(11.08)$ & $1.03(3.27)$ & $11.56(8.83)$ \\
\hline & R2D1 & $6.67(5.24)$ & $8.09(11.40)$ & $-11.79(8.07)$ & $5.36(9.65)$ & $-0.48(4.98)$ & $12.96(10.12)$ \\
\hline & R1D2 & $7.57(5.04)$ & $8.63(10.64)$ & $-12.19(8.66)$ & $4.73(10.82)$ & $1.76(4.67)$ & $9.36(8.25)$ \\
\hline & R2D2 & $7.60(5.20)$ & $6.36(7.48)$ & $\begin{array}{l}-10.93(8.83) \\
\end{array}$ & $2.73(10.07)$ & $1.14(4.21)$ & $11.97(9.04)$ \\
\hline & R1D3 & $7.23(6.18)$ & $7.37(9.89)$ & $-11.94(7.61)$ & $3.26(11.68)$ & $1.51(3.58)$ & $11.65(9.78)$ \\
\hline & R2D3 & $7.17(5.90)$ & $8.53(9.51)$ & $-11.63(8.43)$ & $3.35(8.75)$ & $0.50(3.80)$ & $14.55(10.08)$ \\
\hline \multirow{6}{*}{ STPF } & R1D1 & $13.72(7.40)$ & $2.08(8.35)$ & $-13.43(7.65)$ & $-12.28(10.74)$ & $8.74(3.74)$ & $4.26(9.26)$ \\
\hline & R2D1 & 13.09(7.76) & $0.89(9.53)$ & $-12.70(7.80)$ & $-9.31(8.36)$ & $8.89(3.24)$ & $5.41(10.47)$ \\
\hline & R1D2 & $13.75(7.04)$ & $2.33(8.07)$ & $-13.54(8.91)$ & $-11.04(10.52)$ & $9.42(5.21)$ & $2.60(8.75)$ \\
\hline & R2D2 & $13.48(7.03)$ & $2.58(5.07)$ & $-11.98(8.28)$ & $-11.35(10.38)$ & $9.19(4.75)$ & $4.29(9.81)$ \\
\hline & R1D3 & $13.22(8.17)$ & $1.49(7.36)$ & $\begin{array}{l}-13.15(7.05) \\
\end{array}$ & $-12.30(9.88)$ & $10.01(6.03)$ & $4.34(10.04)$ \\
\hline & R2D3 & $12.93(8.64)$ & $3.14(6.81)$ & $-12.79(8.06)$ & $-11.91(6.54)$ & $9.36(4.36)$ & $6.97(10.27)$ \\
\hline \multirow{6}{*}{ STPE } & R1D1 & $11.43(4.62)$ & $3.19(7.34)$ & $-13.25(7.84)$ & $-14.63(9.71)$ & $9.90(4.32)$ & $2.54(8.98)$ \\
\hline & R2D1 & $10.68(5.03)$ & $4.42(8.01)$ & $-12.97(7.98)$ & $-11.09(9.69)$ & $9.14(3.36)$ & $3.82(10.88)$ \\
\hline & R1D2 & $10.56(3.93)$ & $3.01(7.01)$ & $-13.67(9.07)$ & $-14.33(9.64)$ & $10.07(4.65)$ & $1.04(8.54)$ \\
\hline & R2D2 & $11.09(4.16)$ & $3.17(5.10)$ & $-12.27(8.51)$ & $-14.20(11.62)$ & $8.99(4.73)$ & $3.67(10.01)$ \\
\hline & R1D3 & $10.43(4.57)$ & $2.13(6.14)$ & $-13.18(6.93)$ & $-15.35(8.73)$ & $10.17(5.46)$ & $2.83(9.98)$ \\
\hline & R2D3 & $10.66(6.12)$ & $4.23(6.36)$ & $-13.07(7.94)$ & $-14.15(6.81)$ & $9.33(5.02)$ & $5.62(10.19)$ \\
\hline \multirow{6}{*}{ SWPF } & R1D1 & $68.86(5.37)$ & $-3.66(8.34)$ & $-12.91(9.35)$ & $6.69(5.58)$ & $5.37(4.23)$ & $2.31(12.04)$ \\
\hline & R2D1 & $68.49(5.13)$ & $-2.01(10.96)$ & $-10.29(7.54)$ & $7.83(9.39)$ & $4.10(4.59)$ & $3.52(16.61)$ \\
\hline & R1D2 & $69.20(4.46)$ & $-4.05(8.74)$ & $\begin{array}{l}-13.44(9.96) \\
\end{array}$ & $8.37(6.34)$ & $5.32(4.17)$ & $2.96(13.75)$ \\
\hline & R2D2 & $68.62(4.82)$ & $-3.39(10.13)$ & $-10.27(8.95)$ & $5.72(7.29)$ & $4.84(3.83)$ & $2.17(15.04)$ \\
\hline & R1D3 & $67.00(5.87)$ & $\begin{array}{l}-3.78(7.78) \\
\end{array}$ & $\begin{array}{l}-13.04(8.91) \\
\end{array}$ & $5.91(8.17)$ & $5.98(3.40)$ & $3.65(14.36)$ \\
\hline & R2D3 & $69.44(5.03)$ & $-3.43(8.48)$ & $-11.44(9.56)$ & $4.98(7.11)$ & $4.98(5.25)$ & $5.93(15.93)$ \\
\hline \multirow{2}{*}{ ROM } & R1D1 & $62.13(7.59)$ & $22.07(7.78)$ & $10.99(2.94)$ & $27.60(6.93)$ & $17.26(5.17)$ & $21.85(3.90)$ \\
\hline & R2D1 & $62.97(6.91)$ & $21.11(6.42)$ & $10.96(1.78)$ & $24.43(5.26)$ & $17.59(4.40)$ & $21.85(4.28)$ \\
\hline
\end{tabular}




\begin{tabular}{|c|c|c|c|c|c|c|c|}
\hline $\mathbf{1}$ & $\mathbf{2}$ & $\mathbf{3}$ & $\mathbf{4}$ & $\mathbf{5}$ & $\mathbf{6}$ & $\mathbf{7}$ & $\mathbf{8}$ \\
\hline \multirow{4}{*}{$\mathrm{ROM}$} & R1D2 & $62.92(6.30)$ & $23.10(6.90)$ & $11.35(2.59)$ & $27.82(6.15)$ & $18.19(4.59)$ & $21.51(4.12)$ \\
\cline { 2 - 8 } & R2D2 & $62.19(6.25)$ & $21.41(5.91)$ & $11.03(1.98)$ & $25.95(7.90)$ & $17.33(4.90)$ & $22.02(4.12)$ \\
\cline { 2 - 8 } & R1D3 & $61.03(8.59)$ & $21.03(7.75)$ & $10.61(2.86)$ & $27.22(5.10)$ & $18.88(3.45)$ & $21.53(4.63)$ \\
\cline { 2 - 8 } & R2D3 & $63.84(6.22)$ & $23.09(7.30)$ & $11.34(1.94)$ & $25.97(6.06)$ & $18.07(4.41)$ & $22.03(3.98)$ \\
\hline
\end{tabular}

${ }^{\text {a }}$ Values are expressed as means (SD), FE - flexion-extension rotation, VV - varus-valgus rotation, IE - internal-external rotation, $\mathrm{AP}$ - anterior-posterior translation, $\mathrm{ML}$ - medial-lateral translation, DP - distal-proximal translation, IC - initial contact, STPF and STPE indicate the peak flexion and peak extension during stance phase, respectively SWPF indicates the peak flexion during swing phase. R1D1 refers to the evaluation on day1 by rater1; R1D2 refers to the evaluation on day2 by rater1; R1D3 refers to the evaluation on day3 by rater1; R2D1 refers to the evaluation on day1 by rater2; R2D2 refers to the evaluation on day2 by rater2; R2D 3 refers to the evaluation on day 3 by rater 2 .

(a)

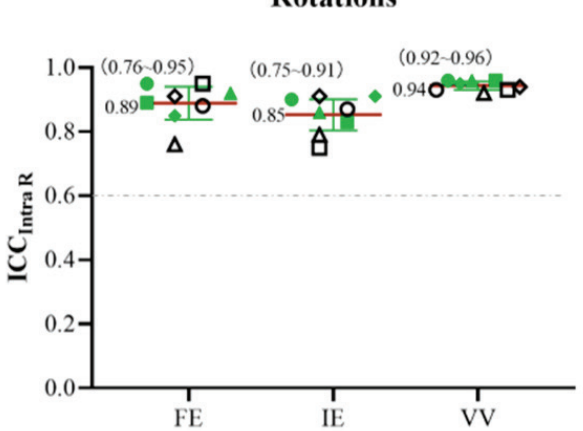

(b)

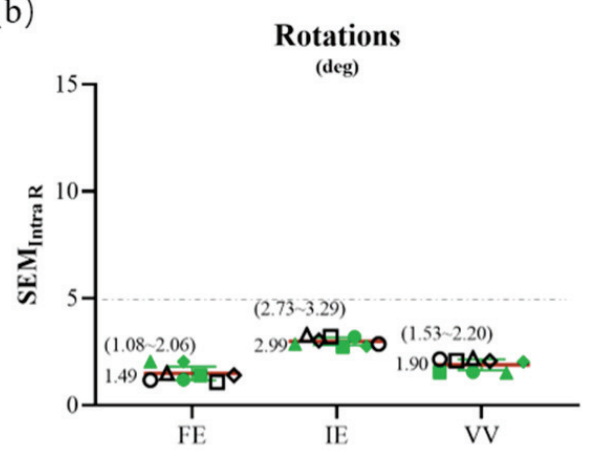

Translations

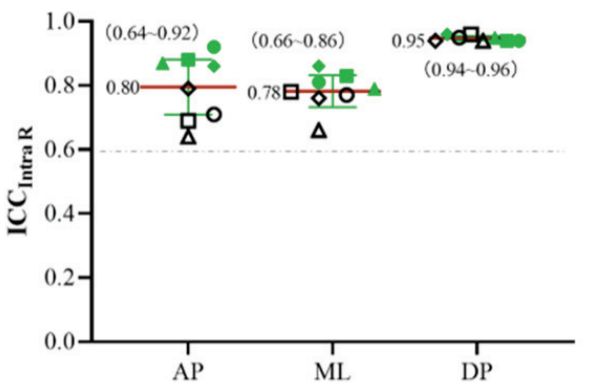

- IC_RI

- IC_R2

A STPF_RI

$\Delta$ STPF_R2

- STPE_R1

口 STPE_R2

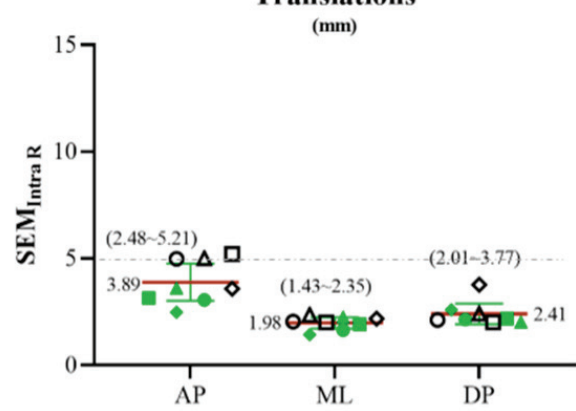

- SWPF_RI

- SWPF R2

Fig. 4. Intra-rater repeatability of motion parameters at four key points during gait cycle:

(a) ICC values, (b) SEM values. For each degree of freedom, the mean (range) of the ICC values are shown. The green represents intra-rater1 repeatability, while the black represents intra-rater2 repeatability.

The circle, triangle, square, and diamond refer to IC, STPF, STPE, and SWPF, respectively

Rotations Translations

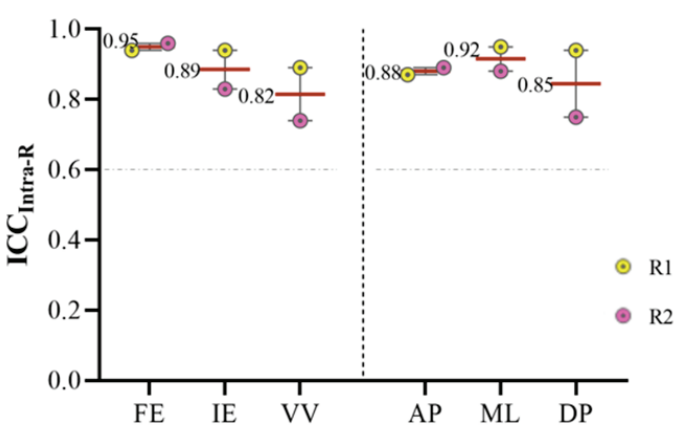

(a)
Rotations(deg) Translations(mm)

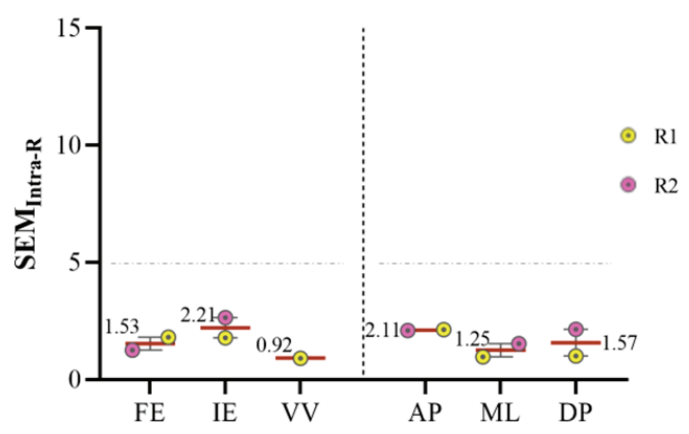

(b)

Fig. 5. Intra-rater repeatability for ROM during gait: (a) ICC values, (b) SEM values.

For each degree of freedom, the mean is shown. The yellow represents intra-rater1 repeatability, while the pink represents intra-rater2 repeatability 
STPE, and SWPF, the mean $\mathrm{SEM}_{\text {Intra } \mathrm{R}}$ values were $1.49^{\circ}, 2.99^{\circ}$, and $1.90^{\circ}$ for $\mathrm{FE}, \mathrm{IE}$, and $\mathrm{VV}$ rotations, respectively, and $3.89 \mathrm{~mm}, 1.98 \mathrm{~mm}$, and $2.41 \mathrm{~mm}$ for AP, ML, and DP translations, respectively (Fig. 4b). For the 6-DOF ROM during the gait cycle, the mean of the $\mathrm{SEM}_{\text {Intra } \mathrm{R}}$ values were $1.53^{\circ}, 2.21^{\circ}$, and $0.92^{\circ}$ for $\mathrm{FE}$, IE, and $\mathrm{VV}$ rotations, respectively, and $2.11 \mathrm{~mm}$, $1.25 \mathrm{~mm}$, and $1.57 \mathrm{~mm}$ for AP, ML, and DP translations, respectively (Fig. 5b).

The Bland-Altman plots indicated the agreement between three different days for the 6-DOF motion trajectory curves of the knee (Fig. 6) and the ROM (Fig. 7) during the gait cycle. The mean and 95\% LoA were calculated based on the motion trajectory parameters of all three days. All of the zero values were in the $95 \%$ LoA. For intra-rater repeatability, the systematic errors were $0.06-1.32^{\circ}$ for rotation and $0.12-1.80 \mathrm{~mm}$ for translation; $96.9 \%$ and $94.2 \%$ of the dots were inside the range of $95 \%$ LoA for the normalized gait cycle and the ROM plots, respectively. This indicated that the agreement of the system was well within raters.

(a)
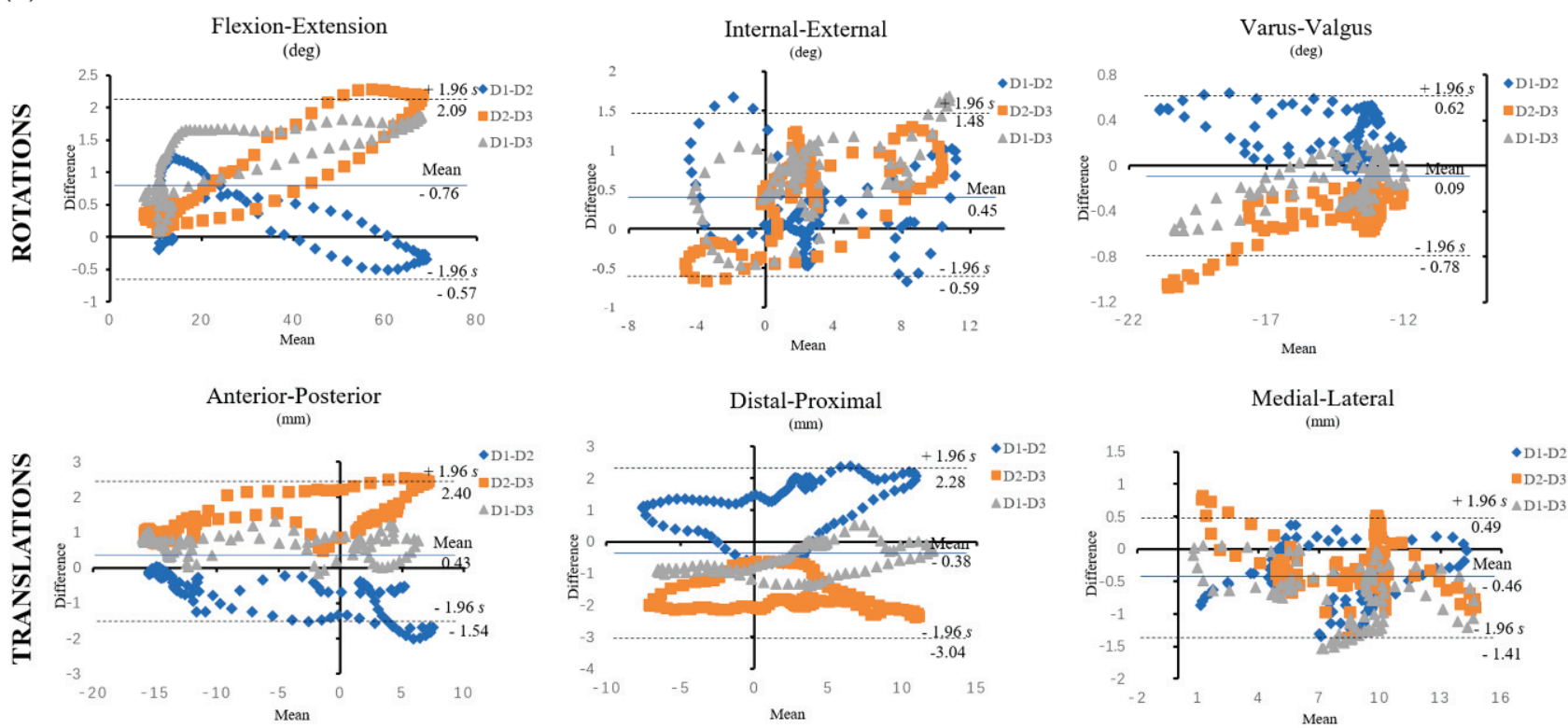

(b)
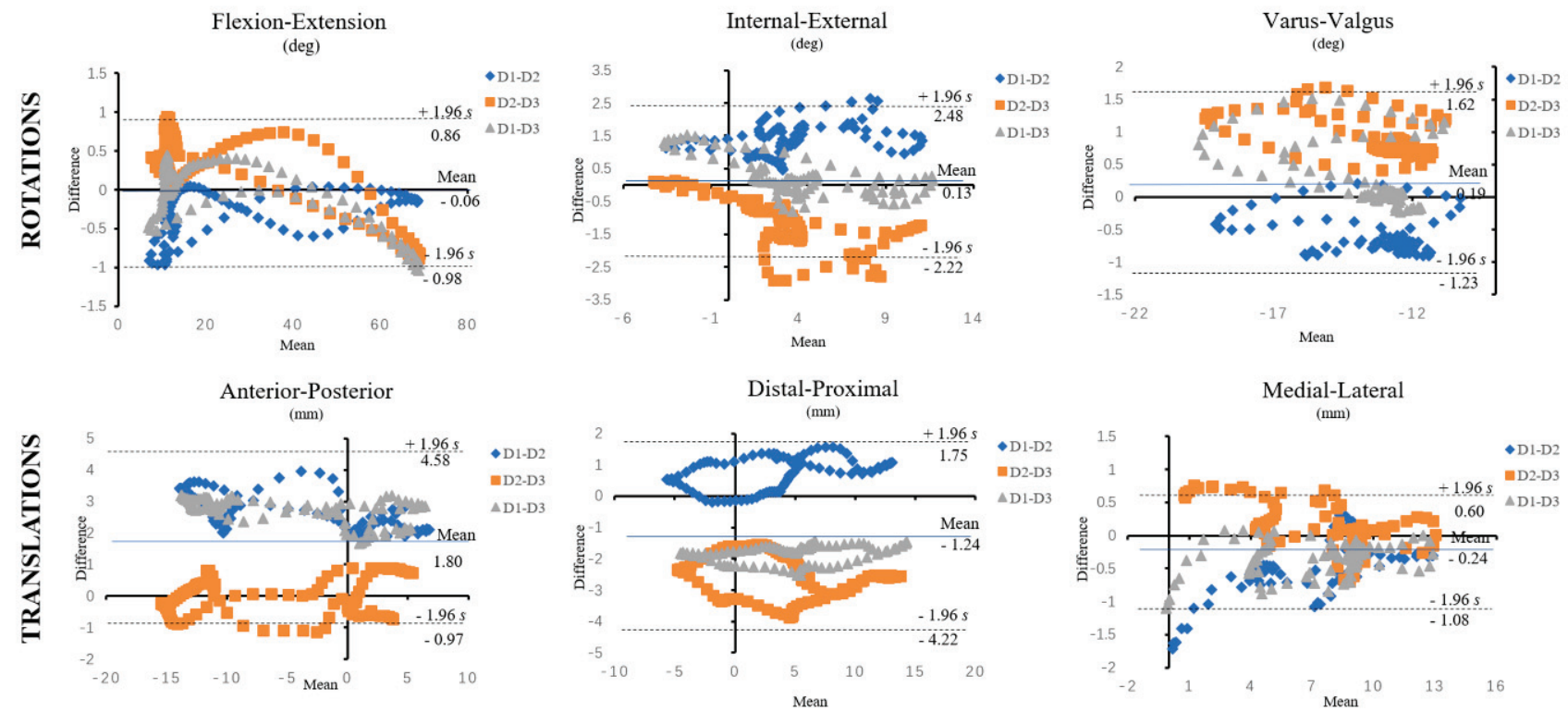

Fig. 6. The Bland-Altman plots of the difference between days against the mean value during gait cycle:

(a) for rater1, and (b) for rater2. The mean and $95 \%$ LoA were based on the average of all three days.

The blue diamond represents the repeatability between day1 and day2 (D1-D2). The orange square represents the repeatability between day2 and day3 (D2-D3). The grey triangle represents the repeatability between day1 and day3 (D1-D3) 
(a)
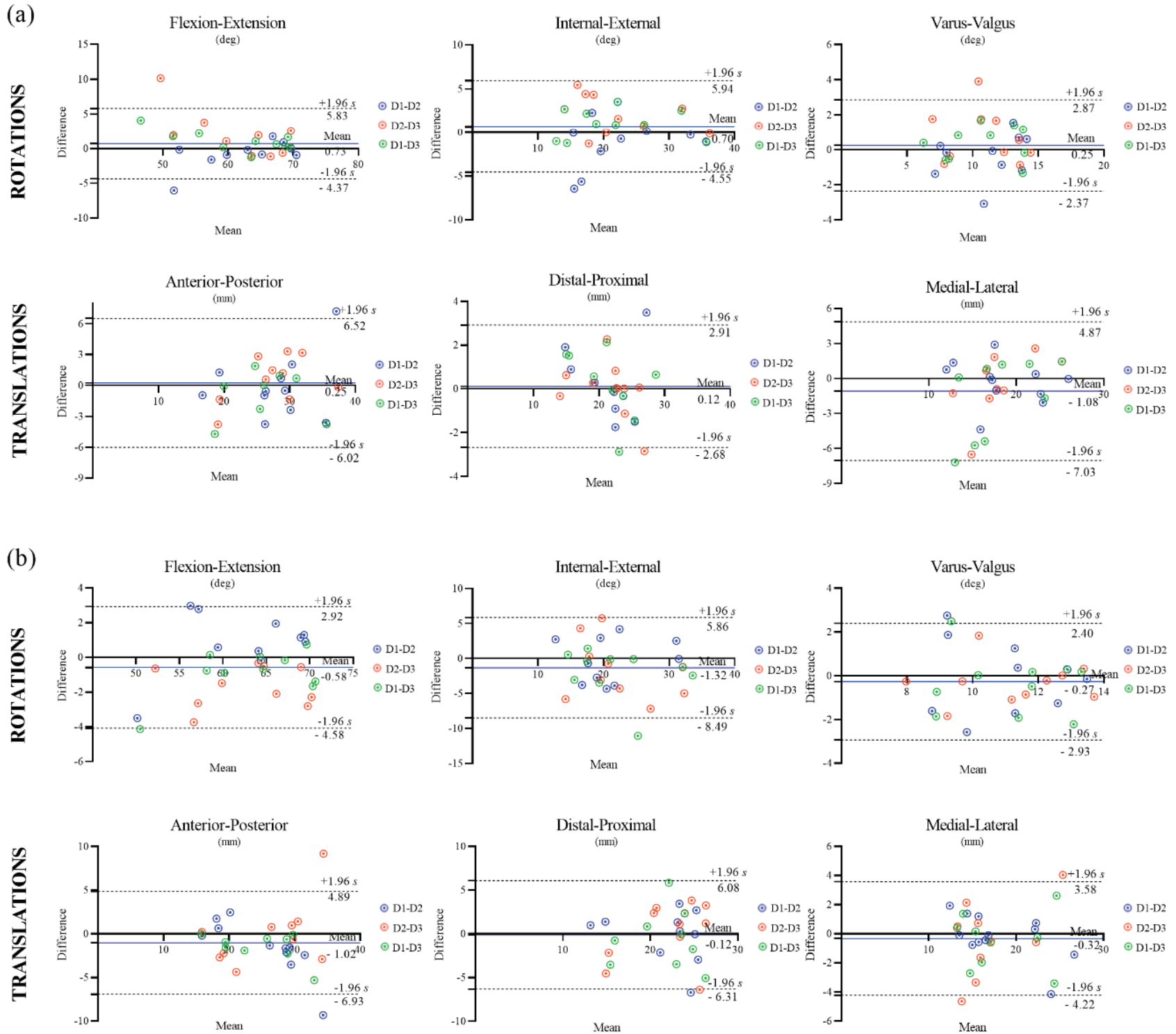

Fig. 7. The Bland-Altman plots of ROM difference between days against the mean value: (a) is for rater1, and (b) is for rater2. The mean and $95 \%$ LoA were based on the average of all three days. The blue represents the repeatability between day1 and day2 (D1-D2). The red represents the repeatability between day2 and day3 (D2-D3). The green represents the repeatability between day1 and day3 (D1-D3)

\subsection{Inter-rater repeatability}

The $\mathrm{ICC}_{\text {Inter-rater }}$ values showed good to excellent correlations for rotation and fair to excellent correlations for translation (Figs. 8a, 9a). From 90 ICC values, $43(95.6 \%)$ for rotations and $37(82.2 \%)$ for translations showed values $\geq 0.70 ; 2(4.4 \%)$ for rotations and $6(13.3 \%)$ for translations showed values $0.61 \sim 0.69$ (included); the lowest 2 (4.4\%) were 0.57 and 0.58 for translation. For the 6-DOF motion trajectory of the knee at IC, STPF, STPE, and SWPF, the mean $\mathrm{ICC}_{\text {Inter-rater }}$ values were $0.87,0.81$, and 0.94 for FE, IE, and VV rotations, respectively, and $0.73,0.73$, and
0.89 for AP, ML, and DP translations, respectively (Fig. 8a). For the ROM of the 6-DOF motion trajectory of the knee, the ICC $_{\text {Inter-rater }}$ values showed good to excellent correlations for rotation and translation

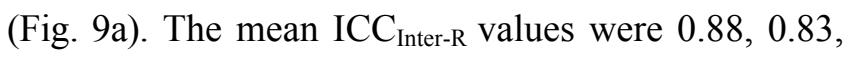
and 0.79 for FE, IE, and VV rotations, respectively, and $0.77,0.84$, and 0.79 for AP, ML, and DP translations, respectively.

The $\mathrm{SEM}_{\text {Inter } \mathrm{R}}$ values are shown in Fig. 8(b) and9(b) to illustrate the inter-rater absolute repeatability. From 90 SEM values, 45 (100\%) for rotations and $42(93.3 \%)$ for translations showed values $\leq 5^{\circ}$ or $5 \mathrm{~mm} ; 3(6.7 \%)$ were $5.55 \mathrm{~mm}, 6.22 \mathrm{~mm}$, and 6.00 $\mathrm{mm}$ for inter-day 1 for AP translations; 34 (75.6\%) for 
rotations and $22(48.9 \%)$ for translations showed values $<3^{\circ}$ or $3 \mathrm{~mm}$. For the 6 -DOF motion trajectory at IC, STPF, STPE, and SWPF, the mean SEM Intra R values were $1.77^{\circ}, 3.33^{\circ}, 1.97^{\circ}$ for $\mathrm{FE}, \mathrm{IE}, \mathrm{VV}$ rotations, respectively, and $4.61 \mathrm{~mm}, 2.17 \mathrm{~mm}$, and $3.56 \mathrm{~mm}$ for AP, ML, and DP translations, respectively (Fig. 8b). For the 6-DOF ROM during the gait cycle, the mean of the $\mathrm{SEM}_{\text {Intra } \mathrm{R}}$ values were $2.38^{\circ}, 2.78^{\circ}$, and $1.07^{\circ}$ for $\mathrm{FE}, \mathrm{IE}$, and VV rotations, respectively, and $3.00 \mathrm{~mm}$, $1.72 \mathrm{~mm}$, and $1.92 \mathrm{~mm}$ for AP, ML, and DP translations (Fig. 9b). Considering less than 50\% SEM values as $<3 \mathrm{~mm}$ for translation, it was suggested that the repeatability between raters for translation requires cautious interpretation.

(a)
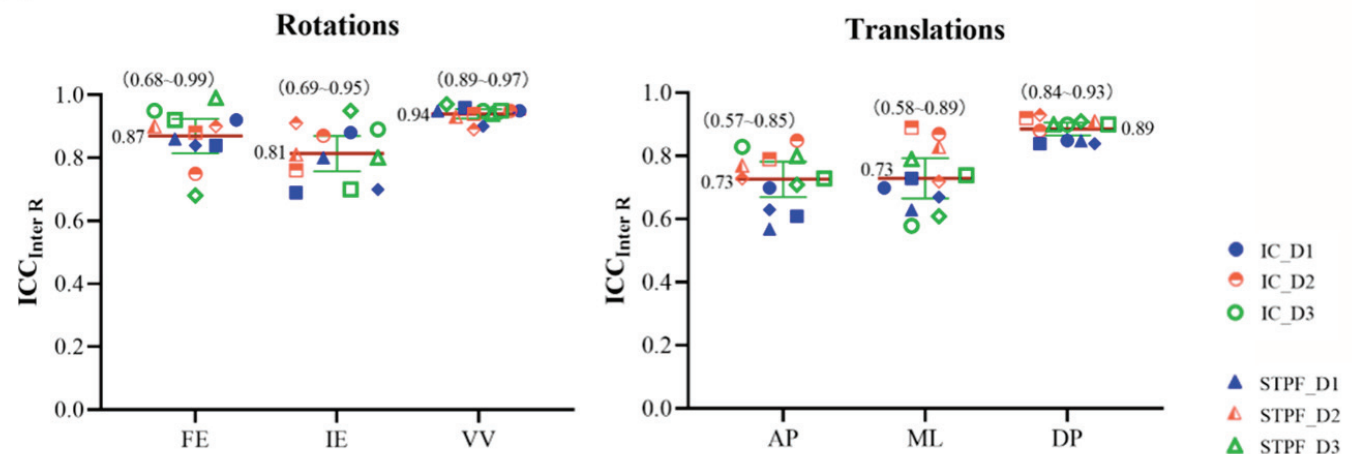

(b)
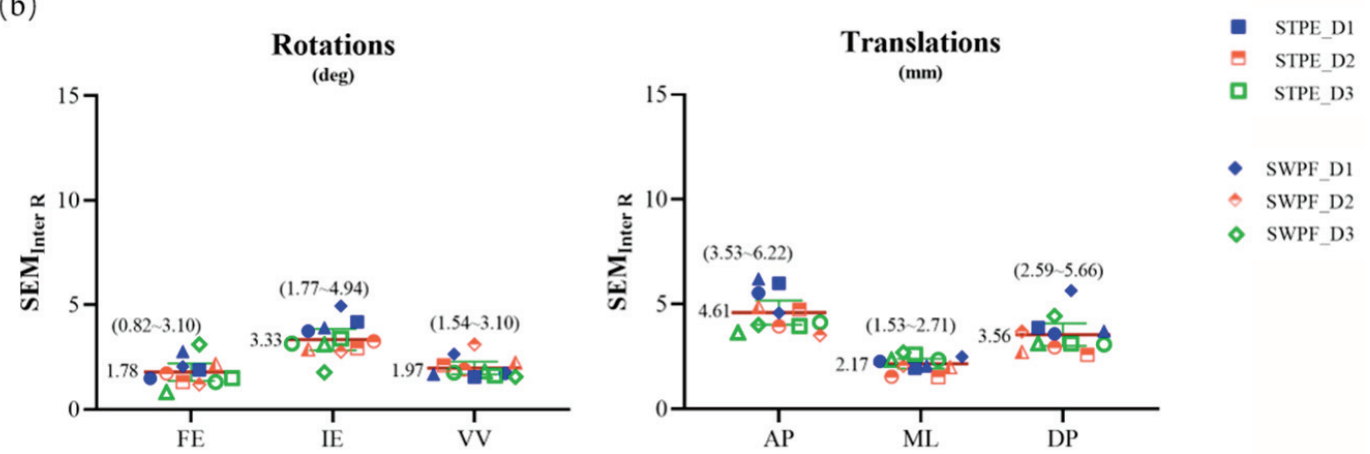

Fig. 8. Inter-rater repeatability of motion parameters at four key points: (a) ICC values, (b) SEM values. For each degree of freedom, the mean (range) is shown. The circle, tringle, square, and diamond refer to IC, STPF, STPE, and SWPF, respectively. The blue, red, and green refer to the inter-rater repeatability on day 1 , day 2 , and day 3 , respectively

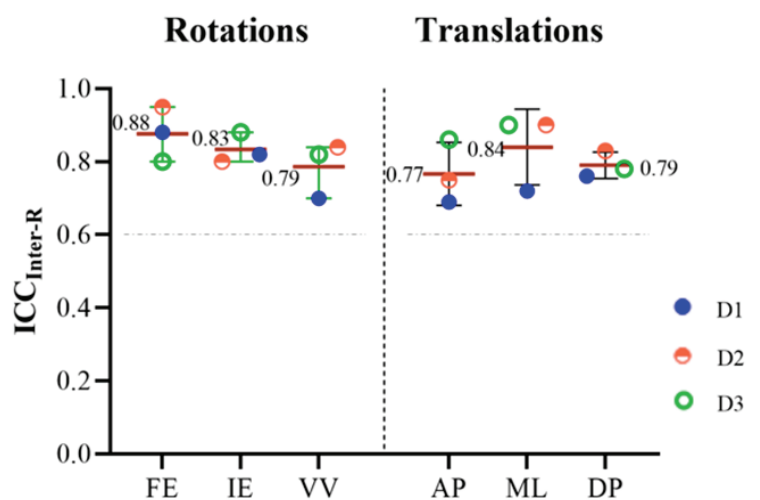

(a)

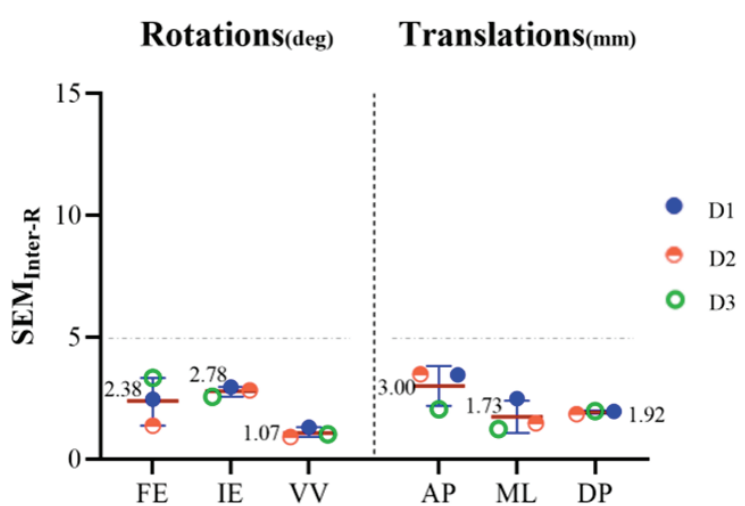

(b)

Fig. 9. Inter-rater repeatability for ROM during gait: (a) ICC values, (b) SEM values.

For each degree of freedom, the mean is shown. The blue, red, and green refer to the inter-rater repeatability on day 1 , day 2 , and day 3 , respectively 


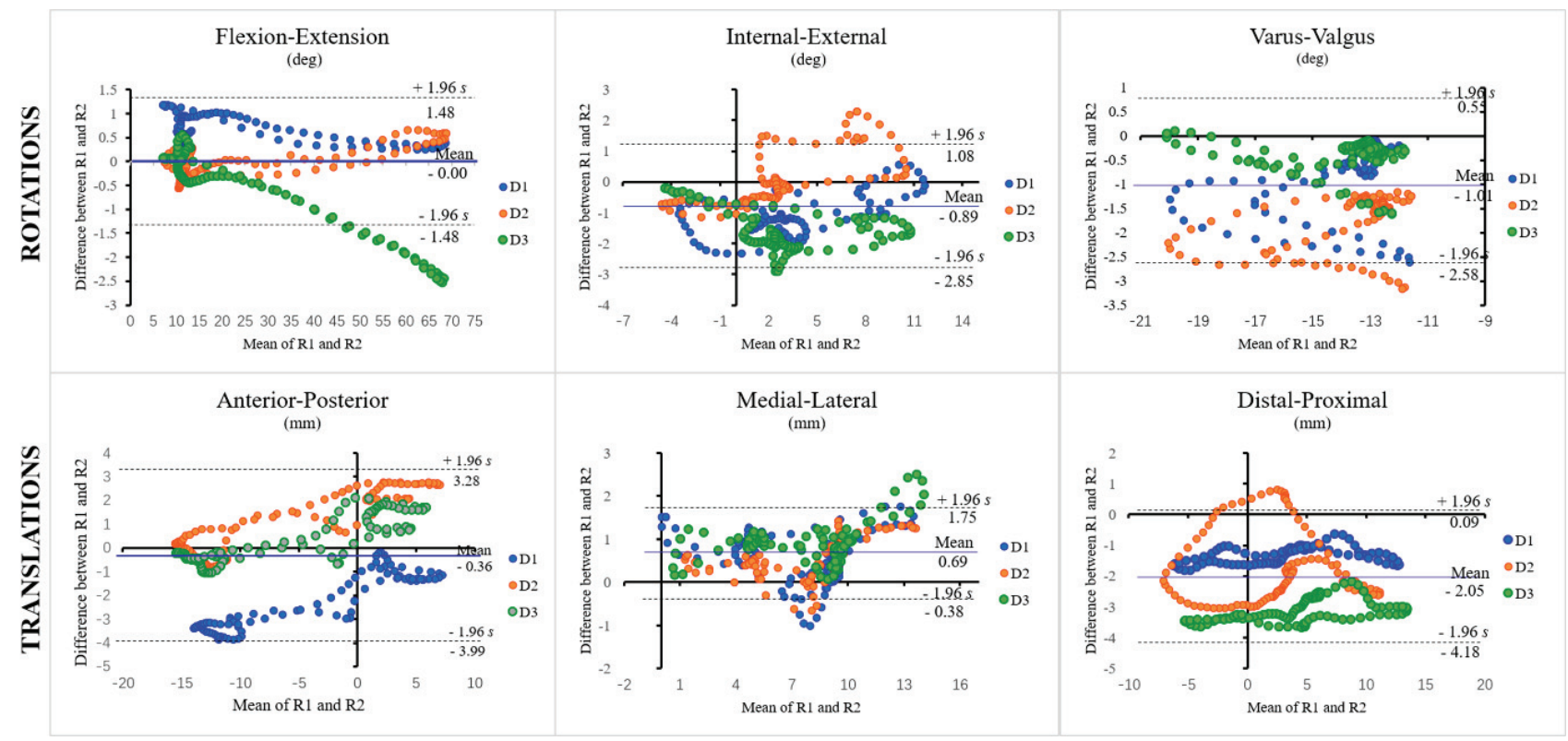

Fig. 10. The Bland-Altman plots of the difference between raters against the mean during gait cycle.

The mean and 95\% LoA are based on the average of all three days. The blue, red, and green refer to the inter-rater repeatability on day 1 , day 2 , and day3, respectively
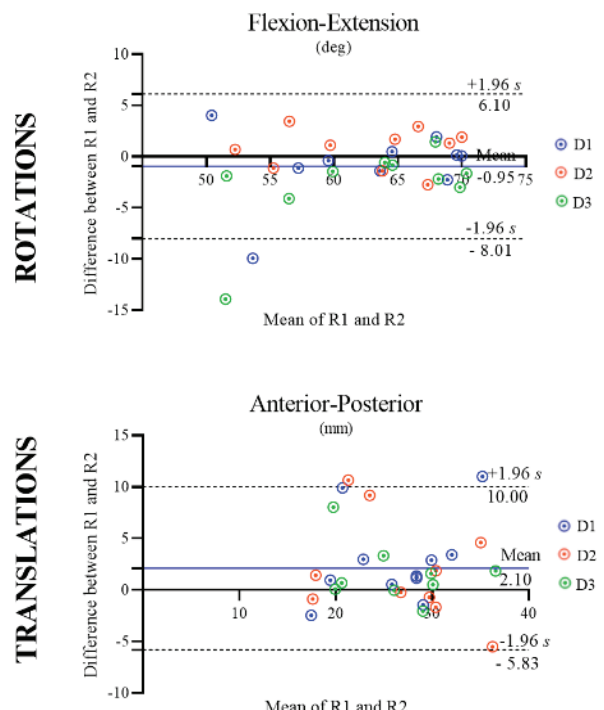
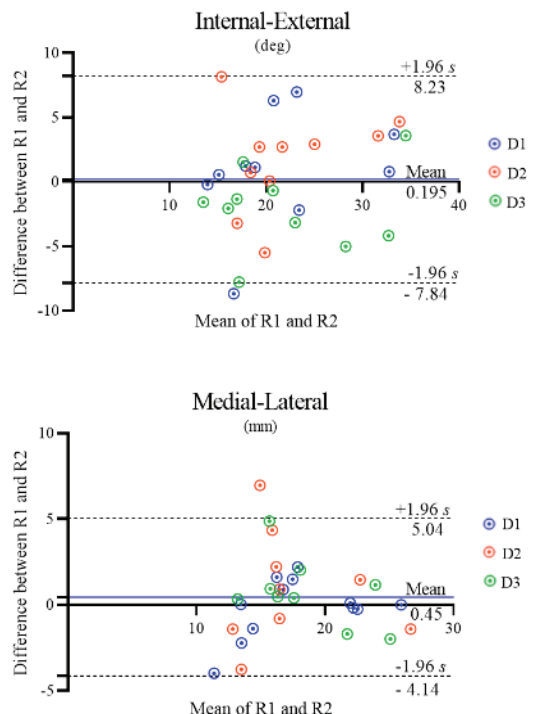
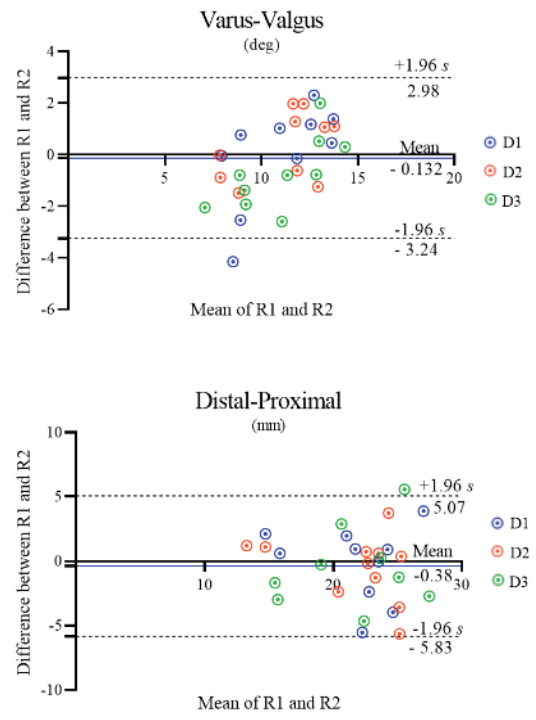

Fig. 11. The Bland-Altman plots of the ROM difference between raters against the mean value. The mean and $95 \%$ LoA are based on the average of all three days. The blue, red, and green refer to the inter-rater repeatability on day 1 , day 2 , and day3, respectively

The Bland-Altman plots indicated the agreement between raters in three days for the 6-DOF motion trajectory curves of the knee (Fig. 10) and ROM (Fig. 11) during the gait cycle. The mean and $95 \%$ LoA were calculated based on the motion trajectory parameters of all three days. All of the zero values were in the 95\% LoA. For inter-rater repeatability, the systematic errors were $0-1.01^{\circ}$ for rotation and $0.38-2.10 \mathrm{~mm}$ for translation; $94.9 \%$ and $95.6 \%$ of the dots were inside the range of $95 \%$ LoA for the normalized gait cycle and the ROM plots, respectively.

\section{Discussion}

This study quantified the intra-rater (between days) and inter-rater (between raters) repeatability for the 
6-DOF motion trajectory of the knee, based on a novel, portable MCS. The results mostly supported our hypotheses. As expected, the intra-rater repeatability for rotation and translation was considered acceptable, as was the inter-rater repeatability for rotation. The intrarater repeatability was better than the inter-rater repeatability. For both intra- and inter-rater repeatability, knee rotation was better than the knee translation. Contrary to what we expected, the inter-rater repeatability for translation requires a cautious interpretation, as less than $50 \%$ SEM values were $\leq 3.0 \mathrm{~mm}$.

For the intra-rater repeatability (between days) on knee rotation, this study showed good to excellent repeatability (Figs. 4(a), 5(a)), which was comparable to or better than the published values for classic MCS [1], [6], [10], [11]. At the key points of motion trajectory in FE rotation, the ICC values in the current study were $0.84 \sim 0.95$ for intra-rater1 repeatability, while the SEM values were $1.21 \sim 2.06^{\circ}$. Based on the VICON system, Bates et al. [1] showed ICC values for FE rotation during gait for hypermobile people to be $0.72 \sim 0.82$ at the key points of motion trajectory. Similarly, using the VICON system, Horsak et al. [10] reported the $\operatorname{ICC}(2, \mathrm{k})$ values as $0.67 \sim 0.87$ and SEM values as $2.41^{\circ} \sim 3.03^{\circ}$ at the key points of motion trajectory for FE rotation during gait for young obese people. In addition, for ROM, the studies showed that ICC values for FE, VV, and IE rotations were 0.66 , 0.40 , and 0.61 [1], respectively, and $0.77,0.96$, and 0.89 [10], respectively. Compared to their results, the ICC values in our study for ROM for FE, VV, and IE rotations were $0.95,0.82$, and 0.89 , respectively, and SEM values were $1.53,0.92$, and $2.21^{\circ}$, respectively. Thus, the repeatability in the current study was better than both of the above studies. Additionally, another study used the Qualisys system and calculated the ICC $(3, \mathrm{k})$ values to be $0.90,0.87$, and 0.85 , and the SEM values to be $1.58,0.90$, and 1.93 for FE, VV, and IE rotations, respectively [11], which were similar values to those obtained in our study. Moreover, the Bland-Altman plots further supported the intra-rater repeatability for rotation with acceptable system errors $\left(\sim 1^{\circ}\right)$. Therefore, based on the portable, optical MCS, the intrarater rotation of the knee joint (between days) was highly repeatable.

Meanwhile, the inter-rater repeatability for rotation was also good to excellent in our study (Figs. 8a, 9a). For the $3 \mathrm{D}$ rotation, $95.6 \%$ of the ICC values were $\geq 0.70$, while $100 \%$ of the SEM values were less than $5^{\circ}$. Nevertheless, the agreement of IE rotation was lower than the FE and VV rotations for both of intra- and inter-rater repeatability, which supported the results of previous study [16]. It was considered the lowest repeatability in the transverse plane, which may be partly caused by very small movements [16]. In addition, the majority of ICC values for inter-rater repeatability were slightly lower than the values for intra-rater repeatability, which was in agreement with previous studies [14]. According to the previous studies, the measurement repeatability may be influenced by the expertise and professional background of the evaluator [16]. In our study, one of the raters (Zheng) was a physiotherapist, while the other (Huang) was not, which may have potentially affected the interrater repeatability. Nevertheless, the inter-rater repeatability for rotation was still approved.

It has still been a challenge to comprehensively evaluate the $3 \mathrm{D}$ translation of the knee joint during movement in vivo. Previous studies observed the testretest repeatability for AP translation, but they detected the AP translation in static conditions using KT-1000 [18]. One study reported the 3D motion trajectory of the tibia with respect to the femur during gait and the measurement repeatability [9]. Hagemeister et al. [9] aimed to detect the repeatability of a functional and postural method for $3 \mathrm{D}$ rotation and AP translation during gait using the Optotrack system. Nevertheless, the calculation method for obtaining the repeatability value was different from ours [9]. In our study, similar to rotation, the ICC and SEM values indicated that the intra-rater1 repeatability was generally higher than intra-rater2 repeatability, which may be, in part, due to the different educational background of the raters. Moreover, the majority of ICC values $(90 \%$ for $\geq 0.70)$ and SEM values $(73.3 \%$ for $<3.0 \mathrm{~mm})$ were acceptable for the intra-rater repeatability, while the rate of acceptable SEM values was less than $50 \%$ for interrater repeatability and only $82.2 \%$ of the ICC values were $\geq 0.70$. We considered that the inter-rater repeatability may also be influenced by the different educational backgrounds of the raters. The Bland-Altman plots further illustrated the acceptable systematic errors. Therefore, the repeatability and accuracy for intra-rater was acceptable, while the inter-rater repeatability requires cautious consideration.

There were several limitations of this study. First, the repeatability results can only be applied to healthy, young adults, because the participants in our study were limited to this demographic group. Therefore, other groups should also be assessed to observe the repeatability. Second, the expertise and educational background differences between the raters may affect the reliability of the measurement, especially the interrater reliability. However, in reality, it is difficult to be evaluated by the same rater or raters with completely the same background. Thus, this study adequately re- 
flected the real situation. Third, the coefficient of multiple correlation (CMC) was not used due to controversy from its strong relationship with ROM [1], [10], even though it is usually used in kinematic repeatability studies during gait [12]. Similar to the previous studies [10], [22], we chose maximum and minimum values during the gait cycle for $\mathrm{FE}$ as the key points of the motion trajectory, as well as ROM for the statistical analysis. Fourth, we did not explore the validity of this portable MCS, which is our next step.

\section{Conclusions}

Based on a portable, optical MCS, we reported measurement accuracy and repeatability of 6-DOF motion trajectory on the knee for the first time. The intra- and inter-rater repeatability were good to excellent for rotation. The intra-rater repeatability was acceptable for translation, while the inter-rater repeatability for translation should be considered cautiously. The educational background of the raters may influence the agreement of the measurements. Therefore, it is recommended that the evaluations be performed by the same rater.

\section{Acknowledgements}

This work was supported by National Key Research and Development Program of China [grant numbers 2018YFF0300504], National Natural Science Foundation of China [grant numbers 81572213 and 81702211], Chunhui Plan of Changzhi Medical College, China [grant numbers QDZ201901]. We thank Peter Mittwede, MD, PhD, from Liwen Bianji, Edanz Editing China (www.liwenbianji.cn/ac), for editing the English text of a draft of this manuscript.

\section{References}

[1] Bates A.V., Mcgregor A.H., AlexAnder C.M., Reliability and minimal detectable change of gait kinematics in people who are hypermobile, Gait Posture, 2016, 44, 37-42, DOI: 10.1016/j.gaitpost.2015.11.002.

[2] Brophy R., Silvers H.J., Gonzales T., Mandelbaum B.R., Gender influences: the role of leg dominance in ACL injury among soccer players, Br. J. Sports Med., 2010, 44, 694-697, DOI: $10.1136 / \mathrm{bjsm} .2008 .051243$.

[3] Chang A.H., Chmiel J.S., Moisio K.C., Almagor O., Zhang Y., Cahue S., Sharma L., Varus thrust and knee frontal plane dynamic motion in persons with knee osteoarthritis, Osteoarthritis Cartilage, 2013, 21, 1668-1673, DOI: 10.1016/j.joca.2013.08.007
[4] Cicchetti D.V., Guidelines, Criteria, and Rules of Thumb for Evaluating Normed and Standardized Assessment Instruments in Psychology, Psychol. Assess., 1994, 6, 284-290, DOI: 10.1037/1040-3590.6.4.284.

[5] CicchetTi D.V., Sparrow S.A., Developing criteria for establishing interrater reliability of specific items: applications to assessment of adaptive behavior, Am. J. Ment. Defic., 1981, 86, 127-137, PMID: 7315877.

[6] Ferber R., Mcclay Davis I., Williams D.S. 3rd, LAUGHTON C., A comparison of within- and between-day reliability of discrete $3 D$ lower extremity variables in runners, J. Orthop. Res., 2002, 20, 1139-1145, DOI: 10.1016/ s0736-0266(02)00077-3.

[7] GRoOd E.S., SunTAY W.J., A joint coordinate system for the clinical description of three-dimensional motions: application to the knee, J. Biomech. Eng., 1983, 105, 136-144, DOI: 10.1115/1.3138397.

[8] Guan S., Gray H.A., Schache A.G., Feller J., De Steiger R., PANDY M.G., In vivo six-degree-of-freedom knee-joint kinematics in overground and treadmill walking following total knee arthroplasty, J. Orthop. Res., 2017, 35, 1634-1643, DOI: $10.1002 /$ jor.23466.

[9] Hagemeister N., Parent G., Van De Putte M., St-Onge N., Duval N., DE GuISE J., A reproducible method for studying three-dimensional knee kinematics, J. Biomech., 2005, 38, 1926-1931, DOI: 10.1016/j.jbiomech.2005.05.013.

[10] Horsak B., Pobatschnig B., Baca A., Greber-Platzer S., Kreissl A., Nehrer S., Wondrasch B., Crevenna R., KEILANI M., KRANZL A., Within-assessor reliability and minimal detectable change of gait kinematics in a young obese demographic, Gait Posture, 2017, 54, 112-118, DOI: 10.1016/ j.gaitpost.2017.02.028.

[11] Hutchinson L., Schwartz J.B., Morton A.M., Davis I.S., Deluzio K.J., RaInBow M.J., Operator Bias Errors Are Reduced Using Standing Marker Alignment Device for Repeated Visit Studies, J. Biomech. Eng., 2018, 140, DOI: 10.1115/1.4038358.

[12] LeIGH R.J., PoHL M.B., FerBer R., Does tester experience influence the reliability with which $3 D$ gait kinematics are collected in healthy adults?, Phys. Ther. Sport, 2014, 15, 112-116, DOI: 10.1016/j.ptsp.2013.04.003.

[13] Li G., VAN De Velde S.K., Bingham J.T., Validation of a non-invasive fluoroscopic imaging technique for the measurement of dynamic knee joint motion, J. Biomech., 2008, 41, 1616-1622, DOI: 10.1016/j.jbiomech.2008.01.034.

[14] Manca M., Leardini A., Cavazza S., Ferraresi G., Marchi P., Zanaga E., BenedetTi M.G., Repeatability of a new protocol for gait analysis in adult subjects, Gait Posture, 2010, 32, 282-284, DOI: 10.1016/j.gaitpost.2010.05.011.

[15] Matsas A., TAYlOR N., MCBURNEY H., Knee joint kinematics from familiarised treadmill walking can be generalised to overground walking in young unimpaired subjects, Gait and Posture, 2000, 11, 46-53, DOI: 10.1016/s0966-6362(99)00048-x.

[16] McGinley J.L., BAKer R., Wolfe R., Morris M.E., The reliability of three-dimensional kinematic gait measurements: a systematic review, Gait Posture, 2009, 29, 360-369, DOI: 10.1016/j.gaitpost.2008.09.003.

[17] Monaghan K., Delahunt E., Caulfield B., Increasing the number of gait trial recordings maximises intra-rater reliability of the CODA motion analysis system, Gait Posture, 2007, 25, 303-315, DOI: 10.1016/j.gaitpost.2006.04.011.

[18] Myrer J.W., Schulthies S.S., Fellingham G.W., Relative and absolute reliability of the KT-2000 arthrometer for un- 
injured knees. Testing at 67, 89, 134, and $178 \mathrm{~N}$ and manual maximum forces, Am. J. Sports Med., 1996, 24, 104-108, DOI: $10.1177 / 036354659602400119$.

[19] Sinclair J., Taylor P.J., GReEnhalgh A., Edmundson C.J., Brooks D., HobBs S.J., The test-retest reliability of anatomical co-ordinate axes definition for the quantification of lower extremity kinematics during running, J. Hum. Kinet., 2012, 35, 15-25, DOI: 10.2478/v10078012-0075-8.

[20] Stickley C.D., Presuto M.M., RadZAK K.N., BouRBEAu C.M., HetZler R.K., Dynamic Varus and the Development of Iliotibial Band Syndrome, J. Athl. Train, 2018, 53, 128-134, DOI: 10.4085/1062-6050-122-16.

[21] Teufl W., Miezal M., Taetz B., Frohlich M., Bleser G., Validity, Test-Retest Reliability and Long-Term Stability of Magnetometer Free Inertial Sensor Based 3D Joint Kinematics, Sensors (Basel), 2018, 18, DOI: 10.3390/s18071980.

[22] Tian F., Li N., Zheng Z., Huang Q., Zhu T., Li Q., Wang W., TSAI T.Y., WANG S., The effects of marathon running on threedimensional knee kinematics during walking and running in recreational runners, Gait Posture, 2020, 75, 72-77, DOI: 10.1016/j.gaitpost.2019.08.009.

[23] Vincent K.R., ConRad B.P., Fregly B.J., Vincent H.K., The pathophysiology of osteoarthritis: a mechanical perspective on the knee joint, $\mathrm{Pm} \mathrm{r}, 2012,4, \mathrm{~S} 3-9$, DOI: 10.1016/j.pmrj.2012.01.020.

[24] YaO Z.L., WANG S.B., Zhang Y., HuAng W.H., Shen H.Y., Ma L.M., HuAnG H.Y., XIA H., Clinical Significance of a Novel Knee Joint Stability Assessment System for Evaluating Anterior Cruciate Ligament Deficient Knees, Orthop. Surg., 2016, 8, 75-80, DOI: 10.1111/os.12225.

[25] Zeng X., Ma L., Lin Z., Huang W., Huang Z., Zhang Y., MAO C., Relationship between Kellgren-Lawrence score and $3 D$ kinematic gait analysis of patients with medial knee osteoarthritis using a new gait system, Sci. Rep., 2017, 7, 4080, DOI: 10.1038/s41598-017-04390-5.

[26] Zhang Y., Huang W., Yao Z., Ma L., Lin Z., Wang S., Huang H., Anterior Cruciate Ligament Injuries Alter the Kinematics of Knees With or Without Meniscal Deficiency, Am. J. Sports Med., 2016, 44, 3132-3139, DOI: 10.1177/ 0363546516658026. 